\title{
ROLE OF OREXIN 1 RECEPTOR BLOCKER SB-334867 ON CHANGES OF TRIGLYCERIDE AND CHOLESTEROL METABOLISMINDUCED BY PARADOXICAL SLEEP DEPRIVATION IN ADULT MALE RATS
}

\author{
Hoda M. Moghazy ${ }^{1}$, Ahmed MostafaMahmoud ${ }^{1}$, Ashraf M.A. Ali ${ }^{1}$,Khaled A. \\ Abdel-Sater ${ }^{2}$ \\ 1 Medical Physiology department, Sohag University \\ 2Medical Physiology department, Al-AzharUniversity (Assuit Branch)
}

\begin{abstract}
Background: Sleep deprivation (SD) is a growing hazard through its effects on metabolism.Orexin is involved in regulation of both sleep and metabolism. Work on orexin receptors may explainthe mechanisms of some hazardous effects of SD.

Aim:To test the role of orexin-1 receptor (OX1R)blocker, SB-334867 in changes of triglycerides and cholesterol metabolisminduced by SD.

Method: 72 adult albino rats arranged in 4 equal groups: control, SD, SD-OX1R blocked \&SD-DMSO groups. The 3 SD groups are subjected to 8 days of paradoxical SD using the modified multiple platform method. The OX1R blocked group was injected intraperitoneallydaily with single dose (3 mg/kg/day) of SB-334867 dissolved in $2 \mathrm{ml}$ DMSOand diluted 1:1000. The SD-DMSO group was injected by 2 $\mathrm{ml}$ of DMSO diluted 1:1000. Triglycerides and cholesterol levels weremeasured.

Results: Blood triglyceride levels dropped in all groups subjected to SD after the $1^{\text {st }}$ day while the blood cholesterol level dropped in all groups subjected to SD at the $7^{\text {th }}$ or $8^{\text {th }}$ day. In SD-OX1R blocked group showed less drop in blood triglyceridesthan the other SD groups but statistically non-significant change in cholesterol level.

Conclusion: SDleads to earlier and more dropin blood triglyceridesthan the drop in cholesterol levels. This can be explained by high metabolism during SD with dependence on triglyceride more than cholesterol. OX1R blocker partially reduces the drop of triglyceride not cholesterol level indicating that orexinmay be involved in control of triglyceride metabolism but not cholesterol.
\end{abstract}

\section{Introduction}

There is increasing studies to demonstrate that SD is associated with increased risk of cardiovascular disease including hypertension ${ }^{(3)}$.Night shift workers have a higher risk of diabetesas SD predispose for poor metabolic health by promoting excess caloric intake $^{(4)}$. These adverse sequelae were linked to sleep disturbances, including insufficient sleep, fragmented sleep, circadian dysregulation even without altering total sleep duration ${ }^{(5)}$.
Rapid in dustrialization, newtechnologies as smartphones and lifestyle changes have changed the magnitude of stressors to which our current generation is exposed which if uncontrolled might lead to accelerated aging, immune suppression, heart disease, hypertension \& obesity ${ }^{(1)}$. One of these modern stressors is the sleep deprivation (SD). The average sleep duration has declined over the last few decades that represent a major public health issue ${ }^{(2)}$. 
Medicine under a $12 \mathrm{~h}$ light/dark cycle (light on at 6:00 am). Animals were provided ad libitum \& water throughout the study. Experiments were approved by the Ethical Committee of Animal Experiments, Sohag faculty of medicine and in accordance with the Guide for the Care and Use of Laboratory Animals (8th edition, National Academies Press).

\section{Experimental groups}

Animals were randomly divided into four equal groups $(n=18)$ :

\section{Group I (G I-the control group):}

In this group, each threerats were placed inside a cage $(50 \times 45 \times 30 \mathrm{~cm})$ that contain 7 rectangular platforms specified for standing of rats and filled with sawdust to a level $1 \mathrm{~cm}$ below the upper surface of the 7 rectangular plateforms. Rats in this group were allowed to sleep normally. This group was i.p. injected with $2 \mathrm{ml}$ of saline once daily throughout the 8 days of the experiment.

\section{Group II (GII- the sleep deprived group):}

In this group, rats were subjected to paradoxical SD for 8 successive days using a modified multiple platform method ${ }^{(10)}$. Each three rats were placed inside a similar cages as $\mathrm{G}$ I butwater was added to the bottom of the cage instead of the sawdust. The upper surfaces of the platforms were kept $1 \mathrm{~cm}$ above the surface of the water. Thus, the rats could move around inside the cage by jumping from one platform to another. Upon reaching the paradoxical phase of sleep, rats experience muscle atonia, which leads them to make contact with, or fall into, the water. At that point, they awaken abruptly and repeat the sleep-wake cycle. This group was injected with $2 \mathrm{ml}$ saline i.p. once daily for 8 successive days as $\mathrm{GI}^{(11)}$.

Group III (G III - the sleep deprived-OX1R blocked group, SDOX1R blocked group):
These effects of SD on lipid metabolism are still controversy. While some researchers find drop of triglycerides and cholesterol levelsafter $\mathrm{SD}^{(6)}$ others reporteda rise in their levels ${ }^{(7)}$. Also the mechanisms by which SD affects lipid metabolism are still not clearly understood ${ }^{(5)}$.

Orexin, a newly discovered hypothalamic neurotransmitter, is involved in regulation of sleep ${ }^{(8)}$, the neuroendocrine system \&energy homeostasis $^{(9)}$.

Orexin has 2 types of receptors: orexin-1 receptors (OX1R) and orexin2 receptors $(\mathrm{OX} 2 \mathrm{R})$. The OX1R has poor effect on sleep while OX2R has a major role in promoting wakefulness. So Blocking OX1R by SB-334867 without affecting sleep may affect lipid metabolism giving an explanation for the changes in lipid profile during periods of SD.

So, the hypothesis of this study is, orexin may play a role in the disturbances that accompany SD that will be reflected on health due to its wide roles in regulation of sleep and regulation of different parameters that affected by SD.

\section{Aim of the work:}

- To test the effect of SD onbloodtriglyceride and cholesterol levels.

- To evaluate the hypothesis that orexin is involved in the mechanism by which SD produces these changes.

- To test the efficacy of OX1Rblocker, SB-334867 as a protective agent against changes in triglyceride and cholesterol levels.

\section{Materials and methods}

\section{Animals:}

A total of 72 adult male albino rats, aged 10-12 weeks and weighting 200-300 grams obtained from Animal Facility, Faculty of Science, Sohag University. Rats were housed in Animal Facility, Sohag Faculty of 
injection of $2 \mathrm{ml}$ of DMSO diluted 1:1000 in saline i.p. once daily for 8 successive days.

A blood sample was taken daily at 4:00 p.m. from the lateral tail vein of each rat using $3 \mathrm{ml}$ syringe. The samples were collected in EDTA $(20 \mu \mathrm{L} / \mathrm{ml}$ blood) containing tubes. Blood was centrifuged and plasma was separated and stored at $-20^{\circ} \mathrm{C}$ until the time of biochemical analysis ${ }^{(10)}$.
In this group, rats were subjected to paradoxical SD as G II but with i.p. injection of SB-334867 (OX1R blocker) dissolved in $2 \mathrm{ml}$ DMSO and diluted 1:1000 in saline in a dose of 3 $\mathrm{mg} / \mathrm{kg} /$ day once daily for 8 successive days.

Group IV (G IV - the sleep deprivedDMSO group, SD-DMSO group):

In this group, rats were subjected to paradoxical SD as G II but with i.p.

\begin{tabular}{|l|l|}
\hline Time & Procedure \\
\hline 10 a.m. & Removal of food and starting the fasting. \\
\hline 12 p.m. & $\begin{array}{l}\text { Removal of water at the bottom of the cages of G II, G III \&G IV and } \\
\text { drying the cages allowing rats to sleep freely. }\end{array}$ \\
\hline 4 p.m. & $\begin{array}{l}\text { Collection of blood samples for measuring ofblood triglycerides and } \\
\text { cholesterol. } \\
\text { Adding the food after weighting it. } \\
\text { Start of SDby filling the cages with water to a level of 1 cm below the } \\
\text { upper surfaces of the rectangular platforms in all groups except G I. } \\
\text { Injection of rats in G I \& G II with saline, rats of G III with OX1R } \\
\text { blocker i.p.\& rats of G IV with DMSO i.p. }\end{array}$ \\
\hline
\end{tabular}

Table (1): Time schedule details during the 8 days of the experiment

compared with the control group. The fasting triglyceride level in G III was statistically significanthigher when compared with $\mathrm{G}$ II beginning after the $1^{\text {st }}$ day of SD (P-value $\left.<0.0001\right)$ and continued to the $8^{\text {th }}$ day $(\mathrm{P}$-value $=$ 0.0044). The difference was more obvious during the $1^{\text {st }} 4$ days.

\section{Cholesterol levels:}

In the sleep deprived group (G II)\& the SD-DMSO group, there was a statistically significant lower fasting blood cholesterol level appeared at the $8^{\text {th }}$ day of SD when compared with the control group (P-value $=0.0121$ for $\mathrm{G}$ II \&0.0048 for G IV). In SD-OX1R blocked group (G III), there was a statistically significant lower fasting blood cholesterol level beginning at the $7^{\text {th }}$ day of SD $(\mathrm{P}$-value $=0.0199)$ and continued to the $8^{\text {th }}$ day $(\mathrm{P}$-value $=$ 0.0021)when compared with the control group. While, by comparing fasting blood cholesterol level in G III and G II showed no statistically significant difference.
The fasting Level oftriglycerides and cholesterolwere measured from these blood samples using triglyceride kits\& cholesterol kits respectively (S.L., Agappe diagnostics LTD., India).

\section{Statistical analysis}

In this work statistics were done by using prism program, version 7 .

Data expressed at mean \pm SE (standard error).Student t-test was used to determine significance between numeric data between two groups.ANOVA test was used to determine significance between numeric data of different groups.Probability value ( $P$-value) was considered Significant if $\mathrm{P}<0.05$.

\section{Results}

\section{Triglyceride levels:}

In all groups subjected to SD (G II, $G$ III\&G IV), the fasting blood triglyceride level showed a statistically significant lower values beginning afterthe $1^{\text {st }}$ day of SD(P-value $<0.0001$ in all groups) and continued to day 8 (P-value $<0.0001$ in all groups) when 


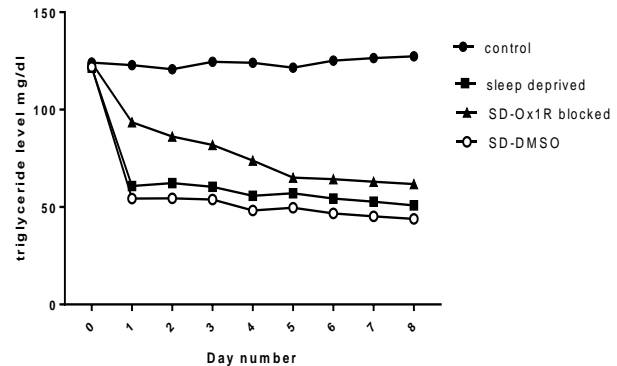

Figure (1): Time course of changes in fasting blood triglyceride level in different groups throughout the experiment.

\section{Discussion}

Our modern society suffers new kinds of stressors; one of them is the SD which has negative effects on different body functions including lipid metabolism. Nevertheless, the role of orexin in health hazards of SD is not yet evident and the mechanisms by which orexin involved in these hazards and the protective effect of orexin receptor blockers are still debated ${ }^{(12)}$.

To accomplish the goals, an animal model of SD was established and the experiment is formed of control group, SD group, SD-OX1R blocked group \& SD-DMSO group. In this study,fasting blood triglyceride\&cholesterol levels were measured and the results were recorded in all groups.

The current study showed that the triglyceride level in the sleep deprived group (G II) and SD-DMSO group (G IV) was lowered to statistically significant values after the $1^{\text {st }}$ day of SD when compared with the control group. while the cholesterol level was statistically significant lower than the control group at the $8^{\text {th }}$ of SD. In the SD-OX1R blocked group (G III), Triglyceride levels in G III showed statistically significant lower values after the $1^{\text {st }}$ day of SD when compared with the control group but the drop in $\mathrm{G}$ III is less than the drop observed in G II which was statistically significant. This suggests a role of orexin in

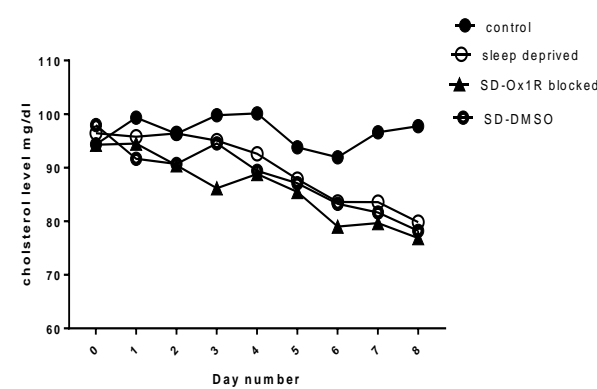

Figure (2): Time course of changes in fasting blood cholesterol level in different groups throughout the experiment.

triglyceride metabolism.

The cholesterol level showed statistically significant lower values at the $7^{\text {th }} \& 8^{\text {th }}$ day of SD with no significant changes when compared with $G$ II denoting this effect on cholesterol is orexin independent.

These results agreed with results of previous studies that reported that SD reduces the triglyceride levels in rats but doesn't affect the cholesterol level $^{(13,14)}$. Plasma concentrations of triglyceride decreased significantly after the $1^{\text {st }}$ day of paradoxical SD but after 8 days of SD, the total cholesterol levels were not modified ${ }^{(10)}$.While, some studies reported a drop in both triglyceride and cholesterol levels in SD rats by lesions of VLPO nucleus ${ }^{(6)}$.On contrast, some studies reported higher triglyceride and cholesterol levels in rats subjected to $\mathrm{SD}^{(7)}$ which may be due to dietetic factor.

The drop in lipid concentration could be a result of high energy expenditure that contributes to energy deficit in sleep deprived rats ${ }^{(10)}$.This maybe explained by stimulation of stress hormones secretion specially thyroxine by orexin-B at the hypothalamic level ${ }^{(15)}$.

\section{conclusion}

SD leads to a rapid and remarkable drop in blood triglyceride levels with a 
slight and delayed drop in blood cholesterol level that can be explained by the high metabolic rate during periods of SD. Blocking OX1R leads to rapid but less remarkable drop in blood triglyceride level but no significant effect on changes of blood cholesterol level.

\section{References}

1. Pressman A, Hernandez A, Sikka SC. Chapter 5 - Lifestyle Stress and Its Impact on Male Reproductive Health. In: Sikka SC, Hellstrom WJG, editors. Bioenvironmental Issues Affecting Men's Reproductive and Sexual Health. Boston: Academic Press; 2018. p. 7383.

2. Youngstedt SD, Goff EE, Reynolds AM, Kripke DF, Irwin MR, Bootzin RR, et al. Has Adult Sleep Duration Declined Over the Last 50+ Years? Sleep medicine reviews. 2016;28:6985.

3. Jeddi S, Ghasemi A, Asgari A, Nezami-Asl A. Role of inducible nitric oxide synthase in myocardial ischemiareperfusion injury in sleep-deprived rats. Sleep and Breathing. 2018;22(2):353-9.

4. McHill AW, Wright KP. Role of sleep and circadian disruption on energy expenditure and in metabolic predisposition to human obesity and metabolic disease. Obesity Reviews. 2017;18(S1):15-24.

5. Koren D, O'Sullivan KL, Mokhlesi B. Metabolic and glycemic sequelae of sleep disturbances in children and adults. Current diabetes reports. 2015;15(1):562.

6. Vetrivelan R, Fuller PM, Yokota S, Lu J, Saper CB. Metabolic effects of chronic sleep restriction in rats. Sleep. 2012;35(11):1511-20.

7. She M, Hu X, Su Z, Zhang C, Yang S, Ding L, et al. Piromelatine, a novel melatonin receptor agonist, stabilizes metabolic profiles and ameliorates insulin resistance in chronic sleep restricted rats. European journal of pharmacology. 2014;727:60-5.

8. Kargar HM-P, Azizi H, MirnajafiZadeh J, Reza MA, Semnanian S.
Microinjection of orexin-A into the rat locus coeruleus nucleus induces analgesia via cannabinoid type-1 receptors. Brain research. 2015;1624:424-32.

9. Villano I, Messina A, Valenzano A, Moscatelli F, Esposito T, Monda V, et al. Basal Forebrain Cholinergic System and Orexin Neurons: Effects on Attention. Frontiers in Behavioral Neuroscience. 2017;11:10.

10.Brianza-Padilla $\mathrm{M}$, Bonilla-Jaime $\mathrm{H}$, Almanza-Pérez JC, López-López AL, Sánchez-Muñoz F, Vázquez-Palacios G. Effects of different periods of paradoxical sleep deprivation and sleep recovery on lipid and glucose metabolism and appetite hormones in rats. Applied Physiology, Nutrition, and Metabolism. 2015;41(3):235-43.

11. White C, Ishii Y, Mendoza T, Upton N, Stasi L, Bray G, et al. Effect of a selective OX1R antagonist on food intake and body weight in two strains of rats that differ in susceptibility to dietary-induced obesity. Peptides. 2005;26(11):2331-8.

12.Tsuneki H, Wada T, Sasaoka T. Chronopathophysiological implications of orexin in sleep disturbances and lifestyle-related disorders. Pharmacology \& therapeutics. 2017.

13. Andersen ML, Martins PJF, D'Almeida V, Santos RF, Bignotto M, Tufik S. Effects of paradoxical sleep deprivation on blood parameters associated with cardiovascular risk in aged rats. Experimental Gerontology. 2004;39(5):817-24.

14.Alvarenga TA, Tufik S, Pires GN, Andersen ML. Influence of food restriction on lipid profile and spontaneous glucose levels in male rats subjected to paradoxical sleep deprivation. Clinics. 2012;67(4):37580.

15.Messina A, De Fusco C, Monda V, Esposito M, Moscatelli F, Valenzano A, et al. Role of the orexin system on the hypothalamus-pituitary-thyroid axis. Frontiers in neural circuits. 2016;10:66. 
SOHAG MEDICAL JOURNAL

Vol. 23 No.2Apr 2019 